\title{
LIFETIMES OF CHARGED AND NEUTRAL D MESONS
}

\author{
The ACCMOR Collaboration

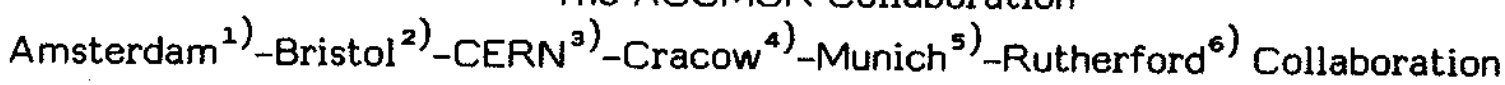

S. Barlag $5, H$. Becker $^{5 D}$, T. Böhringer ${ }^{3} \triangle$, M. Bosman ${ }^{3 \leftrightarrow}$, V. Castillo ${ }^{3}$, V. Chabaud ${ }^{3}$, D. Buchholz 5 , C. Damerell 6 , C. Daum ${ }^{1}$, H. Dietl ${ }^{5}$. H. Fawcett ${ }^{2}$, S. Gill ${ }^{6}$, A. Gillman' ${ }^{6}$, R. Gilmore ${ }^{2}$, T. Gooch ${ }^{2}$, L. Görlich 4 , Z. Hajduk ${ }^{4}$, B.D. Hyams ${ }^{3}$, D.P. Kelsey ${ }^{3}$, J. Kemmer ${ }^{7}$, R. Klanner ${ }^{5}{ }^{7}$, U. Kötz ${ }^{3 \dagger}$, S. Kwan'2, B. Lücking ${ }^{5}$, G. Lütjens 5 , G. Lutz ${ }^{5}$, J. Malos ${ }^{2}$, W. Männer ${ }^{5}$, E. Neugebauer 9 , H. Palka ${ }^{*}$, M. Pepé 6 , J. Richardson ${ }^{6}$, G. De Rijk ${ }^{2 x}$, K. Rybicki ${ }^{4}, H . J$. Seebrunner ${ }^{5}$, U. Stierlin ${ }^{5}$, H.G. Tiecked, M. Turala 4 , G. Waltermann 5 , S. Watts ${ }^{6}$, P. Weilhammer ${ }^{3}$, F. Wickens ${ }^{6}$, L.W. Wiggers ${ }^{2}$, A. Wylies, and T. Zeludziewic $z^{4}$

\begin{abstract}
We have measured the lifetimes of hadronically produced charged and neutral D mesons using silicon microstrip detectors and an active silicon target in the NA32 spectrometer at the CERN SPS. We obtain $\tau_{D^{ \pm}}=\left(10.9 \pm \pm_{1.5}^{1.9}\right) \cdot 10^{-13} \mathrm{~s}$ and $\tau(\bar{D}) 0=$ $(4.2 \pm 0.5) \cdot 10^{-13} \mathrm{~s}$ based on 59 and 90 fully reconstructed decays respectively, giving a ratio $\tau_{D}^{ \pm / \tau}(\bar{D}) \circ$ of $2.6 \pm 0.5$.
\end{abstract}

To be submitted to Zeitschrift für Physik C

1) NIKHEF-H, Amsterdam, The Netherlands

2) University of Bristol, Bristol, UK

3) CERN, Geneva, Switzerland

4) Institute of Nuclear Physics, Cracow, Poland

5) Max Planck Institut für Physik, Munich, Fed. Rep. Germany

6) Rutherford Laboratory, Chilton, Didcot, UK

7) Technische Universität, Munich, Fed. Rep. Germany

ㅁ) Visitor from Gesamthochschule, Saarbrücken, Fed. Rep. Germany

$\triangle$ ) Now at University of Lausanne, Lausanne, Switzerland

$\leftrightarrow$ ) Now at MPI, Munich, Fed. Rep. Germany

§) Visitor from Northwestern University, Evanston, IL, USA

7) Now at DESY, Hamburg, Fed. Rep. Germany

t) Visitor from DESY, Hamburg, Fed. Rep. Germany

(a) Now at Universität - GH Siegen, Siegen, Fed. Rep. Germany

*) Visitor from Institute of Nuclear Physics, Cracow, Poland

\&) Now at Fachhochschule Heilbronn, Fed. Rep. Germany

$x)$ Now at CERN, Geneva, Switzerland 


\section{INTRODUCTION}

Over the past ten years, there have been many efforts to study the production and decay properties of charmed particles in $\mathrm{e}^{+} \mathrm{e}^{-}$annihilation and in neutrino, photon and hadron induced interactions [1]. The relative production rate of charm is smallest ( $\sim 1 / 1000$ of the total cross-section at SPS energies) for hadron-hadron interactions, but the absolute rate of production is highest. Thus, the possibility of collecting high statistics samples of charm has led to many attempts to study charmed particles produced in hadronic interactions [2-4]. The main difficulty in these experiments has been to find a very selective signature to separate the signal from the overwhelming combinatorial background in invariant mass plots. In the NAll experiment [2], we were the first to use a telescope of silicon microstrip detectors to reconstruct charm decay vertices from precisely measured tracks in the environment of a fully electronic experiment. We measured the $(\bar{D}) \circ$ and $D^{ \pm}$ lifetimes from 50 fully reconstructed decays, demonstrating that this new technique is well suited to high statistics hadronic charm production experiments. The same data have been used in addition to extract the lifetime from a sample of 74 semileptonic decays of the charged $D$ into $K^{*}(892) e v_{e}[3]$.

The NA32 experiment is the successor to NAll. In NA32 the Be target was replaced by a finely segmented silicon active target, which enabled us to recognize the interaction point very precisely and also to distinguish between secondary interactions and decays inside the target. A sample of 38 million events has been collected in 1984 using an interaction trigger. We present here results on $(\bar{D}) \circ$ and $D^{ \pm}$ lifetimes based on the full data sample.

\section{EXPERIMENT}

The experiment was performed at the CERN SPS using the ACCMOR spectrometer $[5,6]$ in an unseparated hadron beam of $200 \mathrm{GeV} / \mathrm{c}$ with Cerenkov identification of incident pions and kaons. A schematic layout of the spectrometer is shown in Fig. 1. It consisted of two magnets $(M 1, M 2)$ and 48 planes of drift chambers arranged in four groups (DC2,DC3A,DC3B,DC3C). Three multicellular threshold Cerenkov counters $(\mathrm{C} 1, \mathrm{C} 2, \mathrm{C} 3)$ were used to identify $\pi, K$ and $\rho$ in a wide momentum range extending from about 3 to $90 \mathrm{GeV} / \mathrm{c}$. The main characteristics of these counters are listed in Table 1. 
Figure 2 shows an enlarged view of the target area. The active target consisted of 14 planes of identical silicon microstrip detectors, spaced at $500 \mu \mathrm{m}$ distance along the beam, each plane having 48 horizontal strips of $20 \mu \mathrm{m}$ pitch. The 14 detectors were followed at a distance of $35 \mathrm{~mm}$ by two larger microstrip detectors (multiplicity counters) with a strip pitch of $400 \mu \mathrm{m}$. Each strip was connected via pre- and main-amplifiers to fast ADCs [7]. The characteristics of this arrangement are summarized in Table 2. The detectors were aligned in the plane transverse to the beam with a precision of $\sim 1 \mu \mathrm{m}$.

In order to use the active target in the trigger, speed was of prime importance. For this reason fast linear amplifiers were used. The r.m.s noise at the input was $\sim 3 \cdot 10^{-16} \mathrm{C}$, to be compared to the mean charge of $\sim 4 \cdot 10^{-15} \mathrm{C}$ deposited by a minimum ionizing particle (m.i.p.). The output amplifier-shaper chain provided fast pulses with a full width half maximum of $25 \mathrm{nsec}$. These signals were digitized with an 8-bit ADC with a range of up to 25 m.i.p. and in parallel fed via a discriminator into the trigger logic.

The beam telescope placed in front of the active target consisted of 2 planes of silicon microstrip detectors with capacitive charge division readout $[8,9](60 \mu \mathrm{m}$ readout pitch) and two planes of active target type detectors ( $20 \mu \mathrm{m}$ readout pitch).

The trigger required that a single beam particle go through one of the middle 16 strips of both the last plane of the beam telescope and the first active target plane. An interaction, defined by having at least one strip with a pulse height of 3 m.i.p. or more, had to occur in the central 16 strips of planes 2 to 7 . In addition, a charged particle multiplicity of three or more was required in the two downstream multiplicity counters, this last requirement being made to exclude triggers in the target due to elastic scattering and low multiplicity diffractive interactions.

The measurement of high precision coordinates for reconstructing charm decays was performed by a telescope of 7 silicon microstrip detectors (MSDs) with capacitive charge division readout. The main features of these detectors are summarized in Table 3. With this setup the measurement precision for tracks reconstructed in one of the two detector projections $\left( \pm 14^{\circ}\right)$ and extrapolated to the $z$ position of the primary vertex was about $13 \mu \mathrm{m}$ plus a multiple scattering contribution of $\frac{90}{\mathrm{p}} \mu \mathrm{m}$, where $p$ is the momentum in $\mathrm{GeV} / \mathrm{c}$. 
To best illustrate the functioning of the active target, we show in Fig. 3a a display of an event as seen in the detector. The numbers give the measured ionization loss of the charged particles in the target planes in units of $1 / 10 \mathrm{~m}$.i.p. The beam particle traverses the target until plane 5 where an interaction, marked by a large energy deposit, takes place. Several tracks emerge from the primary vertex, including at least two fragments at large angles. In the forward direction one track traverses the target and is reconstructed in the MSDs and drift chambers (track 2 in Fig. 3b). $A D^{\circ} \rightarrow \mathrm{K}^{-} \pi^{+} \pi^{+} \pi^{-}$decay occurs in the region of plane 12. The four decay tracks are all measured in MSDs and drift chambers (tracks 1,3,4,5). The reconstructed momentum vector of the $D$ is shown as a dotted line in Fig. $3 \mathrm{~b}$. The mass of the D is $1.869 \pm 0.009 \mathrm{GeV} / \mathrm{c}^{2}$.

In the offline analysis the interaction plane was reconstructed on the basis of the measured pulse height in the active target. Using the beam track as a guide, the beam was followed through the active target. The interaction was then detected if a pulse height was encountered larger than 2 m.i.p., followed in the next two consecutive planes by a pulse height larger than $2.5 \mathrm{~m}$.i.p. in windows of respectively 7 and 13 strips around the beam track. The position of the primary vertex in the plane perpendicular to the beam direction was determined by refitting the beam track with the available additional coordinates in the target. We obtained a precision on the primary vertex of $3 \mu \mathrm{m}$ vertically, $25 \mu \mathrm{m}$ horizontally and $80 \mu \mathrm{m}=280 \mu \mathrm{m} / \checkmark 12$, where $280 \mu \mathrm{m}$ is the thickness of an active target plane, along the beam direction independent of the event topology.

\section{DATA ANALYSIS}

Of the total 38 million triggers collected in 1984, about 5 million were taken with incoming $\mathrm{K}^{-}, 22$ million with $\pi^{-}$and 11 million with $\mathrm{p}$. The results presented here are based on the analysis of the $\mathrm{K}^{-}$and $\pi^{-}$data only. In fact charm production from protons is found to be suppressed at a beam momentum of $200 \mathrm{GeV} / \mathrm{c}$, so that including the proton data into our charm sample degrades the signal to background ratio. In a forthcoming publication we will discuss the cross-sections for charm produced with incident $\pi^{-}, \mathrm{K}^{-}$and $\mathrm{p}$.

To reduce the computer time needed for data processing, we preselected events on the basis of the information from the silicon counters only. Events were retained which contained at least two track projections of $\pm 14^{\circ}$ displaced from the primary vertex. The impact parameter cut applied was on average $40 \mu \mathrm{m}$, which has to be compared with impact parameters between 30 and $300 \mu \mathrm{m}$ for particles with a lifetime of $\sim 10^{-13}-10^{-12}$ s. To avoid losses at small distances, we also selected 
events exhibiting an effective jump in the charge particle multiplicity of at least 2 m.i.p. in the active target. Secondary interactions in the target identified by a pulse height of more than 10 m.i.p. in a strip were rejected. This preselection reduced the data by a factor 4.5 .

For the remaining events, a secondary vertex search was performed using the drift chamber tracks to guide the pattern recognition in the MSDs. This search was restricted to those track combinations with a charged kaon candidate in the $\mathrm{K}^{-} \pi^{+}$, $\mathrm{K}^{-} \pi^{+} \pi^{+}$and $\mathrm{K}^{-} \pi^{+} \pi^{-} \pi^{+}$channels (and c.c.) with an invariant mass between 1.7 and $2.0 \mathrm{GeV} / \mathrm{c}^{2}$.

A good particle identification efficiency is crucial for finding charm and for distinguishing $D, D_{s}$ and $\Lambda_{c}$ decays. Using the pulse height information from each cell of the Cerenkov counters, charged particles were assigned a probability of being a pion, a kaon, or a proton [10]. This identification probability depends on the momentum of the particle and may also be degraded by the presence of nearby particles due to the overlap of light in a single mirror. The particle assignment logic, as well as the performance of the Cerenkov counters, has been tested by using the decay products of $K^{*}, \phi, K_{s}^{\circ}$ and $\Lambda$. In the decay channels involving a kaon and one or more charged pions, a good kaon identification efficiency combined with a low probability of misidentifying a pion as a kaon is important to reduce the high combinatorial background. To measure the kaon identification efficiency, we collected $\sim 21,000 \mathrm{~K}^{*}$ decays into $\mathrm{K}^{ \pm} \pi^{\mp}$ and $\sim 8000 \phi$ into $\mathrm{K}^{+} \mathrm{K}^{-}$. We compared the number of events in the mass spectra calculated once by assigning the kaon mass to one partner of the two-particle combination and once by using the Cerenkov identification. The resulting kaon identification efficiency as a function of momentum is shown in Fig. 4. From this experimentally determined efficiency curve, we derived the mean identification efficiency for kaons from Monte Carlo generated $D$ decays selected in the same way as the real events. This efficiency was found to be $\sim 55 \%$. The pion misidentification probability has been measured to be $\sim 3.5 \%$ from a sample of $\sim 5000$ decays $K_{S}^{0} \rightarrow \pi^{ \pm} \pi^{\mp}$.

Even after preselection and particle identification, no charm signals emerged in the inclusive invariant mass plots of all track combinations in the selected channels due to the large combinatorial background. To achieve the rejection factor of $\approx 10^{5}$ needed to obtain a clean sample of fully reconstructed $D$ decays, it was essential to detect the presence of a secondary vertex using the silicon microstrip detectors. We thus applied the following selection criteria : 
- A good fit ( $90 \%$ C.L.) was required to a secondary vertex separated from the primary vertex by more than $2 \mathrm{~mm}$ ( $3 \mathrm{~mm}$ for the charged $D$ ).

- The reconstructed momentum vector of the $D$ had to point back to the primary vertex (90\% C.L.).

- All decay tracks had to miss the primary vertex by more than $1 \sigma$ ( $2 \sigma$ for the charged D) and at least two of them by more than $3 \sigma$, where $\sigma$ was the calculated error on the impact parameter.

The confidence level associated with the first two criteria was reduced to $80 \%$ for the $K \pi \pi \pi$ decays because of the higher combinatorial background.

To further suppress the background for the two-body decay of the $D^{\circ}$, we added a requirement on the angle $\theta_{K}$ between the kaon and the direction of motion of the laboratory in the $D$ rest frame. Kaons from $D$ decays have in fact an isotropic $\cos \theta_{K}$ distribution in the rest frame of the $D$, while the background has a $\cos \theta_{K}$ distribution which peaks at 1 . We have therefore demanded $\cos \theta_{K}<0.8$.

Unlike the readout of the vertex microstrip detectors, every strip of the active target was read out separately, to achieve the best spatial precision and two-particle separation. Good use was made of this for the reconstruction of the beam track up to the primary vertex, which was very well measured in the target. However, the density in the active target of tracks emerging from the primary vertex was often high, making a general use of the coordinates for tracking difficult. The target was instead employed to sample the charge deposition of particles for offline multiplicity analysis. In particular, as an additional selection criterion, the reconstructed pattern of each event was checked for compatibility with the pulse height measured in the active target [6]. The aim of this consistency check was to reject events for which tracks, which were considered as decay tracks, could be attributed to the primary vertex from the pulse height measured in the active target. Fluctuations in the charge deposition due to the tail of the Landau curve were taken into account. This selection based on the active target information resulted in a substantial rejection of background at small decay lengths, without any appreciable loss of good events. This was cross-checked using a high statistics $K_{S}^{\circ}$ sample. We show in Fig. 5 the mass spectra for $K_{S}^{\circ}$ having a decay length below $10 \mathrm{~mm}$ before (Fig. 5a) and after (Fig. 5b) applying such a cut: the signal stays practically the same while the background for this data sample goes down by a factor 2.5 . 


\section{RESULTS}

The invariant mass distributions of the decays satisfying the selection criteria are shown in Fig. 6. Distinct peaks appear on a low background.

In order to extract the lifetimes from the data we had to correct for the acceptance of the selection criteria [2]. The acceptance for each event was approximated by a step function which is zero for $t\left\langle t_{i}^{\min }\right.$ and $\left.t\right\rangle t_{i}^{\max }$, and one for $t_{i}^{\min }<t<t_{i}^{\max }$, where $t_{i}^{\text {min }}$ and $t_{i}^{\text {max }}$ are the minimum and maximum detectable lifetimes for event $i$. The value of $t_{i}^{\text {min }}$ was determined by keeping all parameters of the event fixed, but reducing the decay path until the event was rejected by the selection criteria, while $t_{i}^{\max }$ was given by the distance between the primary vertex and the first plane of the vertex telescope. We performed a combined maximum likelihood fit to the mass and corrected lifetime distributions for the signal and the background, where the background lifetime distribution was assumed to be exponential. For the mass spectrum we assumed a gaussian signal on a linear background, where the width was obtained from the estimated mass resolution for each event. The measurement error on the lifetime $t_{i}$ was also taken into account in the fit. The results of the maximum likelihood fit are summarized in Table 4. For the lifetimes of $D^{ \pm}$and $(\vec{D}) \circ$ and their ratio we find :

$$
\begin{gathered}
\tau(\bar{D}) \circ=(4.2 \pm 0.5) \cdot 10^{-13} \mathrm{~s} \\
\tau_{D^{ \pm}}=\left(10.9 \pm{ }_{1.5}^{1.9}\right) \cdot 10^{-13} \mathrm{~s} \\
\tau_{D^{ \pm / \tau}}\left(\overline{D^{2}}\right) \circ=2.6 \pm 0.5
\end{gathered}
$$

The lifetime distributions of the $D$ candidates, corrected for the minimum observable lifetime, are given in Fig. 7.

Several sources of possible systematic errors have been investigated: a wrong determination of $t_{i}^{\min }$ or $t_{i}^{\max }$ or a non-constant efficiency between them would introduce a bias in the lifetime. Such an effect could take place at the preselection level or at the selection criteria level. The efficiency of the preselection was checked by looking for $K_{s}^{\circ}$ in a sample of events rejected by the preselection. The ratio of the number of $\mathrm{K}_{\mathrm{s}}^{\circ}$ found in the non-preselected versus preselected data turned out to be small $(\sim 7 \%)$ and independent of the decay distance. 
The distribution of flight paths for a sample of $\sim 1000 \mathrm{~K}_{\mathrm{s}}^{0}$ selected with similar criteria as for the $D$ mesons decaying before the first plane in the vertex telescope was compared with the same distribution generated by Monte Carlo (Fig. 8a). In the Monte Carlo we used the $x_{F}$ and $p_{T}^{2}$ distributions observed in the data and simulated the cuts applied to the real events. The two flight path distributions were in good agreement with each other, thus showing that the effects of the selection criteria were well reproduced. In addition, the flat distribution of measured decay length minus minimal acceptable decay length for the $K_{s}^{\circ}$ (Fig. $8 b$ ) showed that we properly correct for the acceptance, with no significant loss or enhancement for short flight paths.

The selection criteria which determine $t_{i}^{\min }$ and $t_{i}^{\max }$ were also varied over $a$ wide range and the lifetime $\tau_{D}$ was calculated for each control sample. The changes of $\tau_{D}$ were in all cases fully compatible with the statistical fluctuations expected from the exclusion of a fraction of events, as cross-checked by Monte Carlo methods.

The pattern recognition efficiency in the silicon microstrip detectors was checked by developing a second reconstruction procedure based on a different strategy and where in addition no use was made of the active target. About $30 \%$ of the data were processed using this alternative approach. A comparison of the $D$ candidates and of $\sim 300 \mathrm{~K}_{\mathrm{s}}^{\circ}$ selected by the two programs showed a very good overlap of the signal events, while background events, which are essentially due to reconstruction problems, were different in the two cases. From this comparison we concluded that the efficiency for reconstruction of secondary decays in the silicon is greater than $95 \%$.

None of the above studies revealed the presence of a bias in our lifetime determination and all the variations found with respect to our quoted values were within the expected statistical fluctuations. We thus conclude that our systematic errors are smaller than the statistical errors.

\section{SUMMARY}

Using an active silicon target and high resolution silicon strip detectors we have measured the lifetimes of charged and neutral $D$ mesons in three fully constrained decay channels, namely $K^{\mp} \pi^{ \pm}, K^{\mp} \pi^{ \pm} \pi^{ \pm}$and $K^{\mp} \pi^{ \pm} \pi^{\mp} \pi^{ \pm}$. Based on 59 and 90 decays respectively we find $\tau_{D^{ \pm}}=\left(10.9 \pm{ }_{1.5}^{2.9}\right) \cdot 10^{-13} \mathrm{~s}$ and $\tau(\bar{D})_{0}=(4.2 \pm 0.5) \cdot 10^{-13} \mathrm{~s}$. For the ratio $\tau_{D} \pm / \tau(\bar{D})$ o we obtain $2.6 \pm 0.5$, these results being compatible with the 1986 Particle Data Group values [11] and with the more recent high statistics results from experiment $\mathrm{E} 691$ at the Fermilab Tagged Photon Spectrometer [12]. 


\section{Acknowledgements}

We wish to thank:

- the staff of the MPI mechanical workshop for designing and constructing the active target, in particular P. Solc for the diligent work in assembling the target;

- L. Hubbeling and P. Jarron (CERN), P. Weißbach and the staff of the MPI electronics workshop for designing and building the electronics;

- R. Hofmann for contributions to the detector fabrication. 


\section{REFERENCES}

[1] M. Gilchriese, Proceedings of the XXIII Int. Conf. on High Energy Physics, 16-23 July 1986, Berkeley, Ed. S.C. Loken, World Scientific.

[2] R. Bailey et al., Z. Phys. C 28 (1985) 357.

[3] H. Palka et al, Z. Phys. C $\underline{35}$ (1987) 151.

[4] M. Aguilar-Benitez et al, CERN/EP 87-38, 20 February 1987, submitted to Physics Letters $B$.

[5] H. Dijkstra et al, Z. Phys. C $\underline{31}$ (1986) 375.

[6] G. de Rijk, Ph.D. thesis, NIKHEF-H, Amsterdam, 1986.

[7] R. Gilmore et al., Nucl. Instrum. Methods $\underline{224}$ (1984) 161.

[8] B.D. Hyams et al., Nucl. Instrum. Methods 205 (1983) 99.

[9] U. Kötz et al., Nucl. Instrum. Methods A235 (1985) 481.

[10] R. Bailey et al., Z. Phys. C $\underline{30}$ (1986) 51.

[11] Particle Data Group, Phys. Lett. 170B (1986) 1.

[12] J.C. Anjos et al., Phys. Rev. Lett. 58 (1987) 626. 
Table 1

The Cerenkov Counters
$\mathrm{Cl}$
C2
C3

Mirror area $\left(\mathrm{cm}^{2}\right)$

$163.5 \times 60$.

$302.5 \times 110$.

$492 . \times 160.1$

\# mirrors

$2 \times 9$

$2 \times 11$

$2 \times 10$

Refractive index $n-1$

$1.5 \cdot 10^{-3}$

$2.9 \cdot 10^{-4}$

$5.8 \cdot 10^{-5}$

$\pi$-threshold (GeV)

2.5

6.3

13.4

K-threshold (GeV)

8.8

22.2

47.2

p-threshold (GeV)

16.7

42.3

89.8

Table 2

The Active Target

$\begin{array}{lcc} & \text { target } & \text { multiplicity counters } \\ \text { \# planes } & 14 & 2 \\ \text { active area }(\mathrm{X} \times \mathrm{Y})\left(\mathrm{mm}^{2}\right) & 32 \times 0.96 & 36 \times 9.6 \\ \text { detector thickness }(\mu \mathrm{m}) & 280 & 280 \\ \text { dist. between detector centres }(\mu \mathrm{m}) & 500 & 1000 \\ \text { \# strips/detector } & 48 & 24 \\ \text { strip pitch (um) } & 20 & 400 \\ \text { precision ( } \mu \mathrm{m}) & 2.8 & 400 / \sqrt{ } 12 \\ \text { strip orientation (with horizontal) } & 0^{\circ} & 0^{\circ}\end{array}$


Table 3

The Vertex Microstrip Detectors

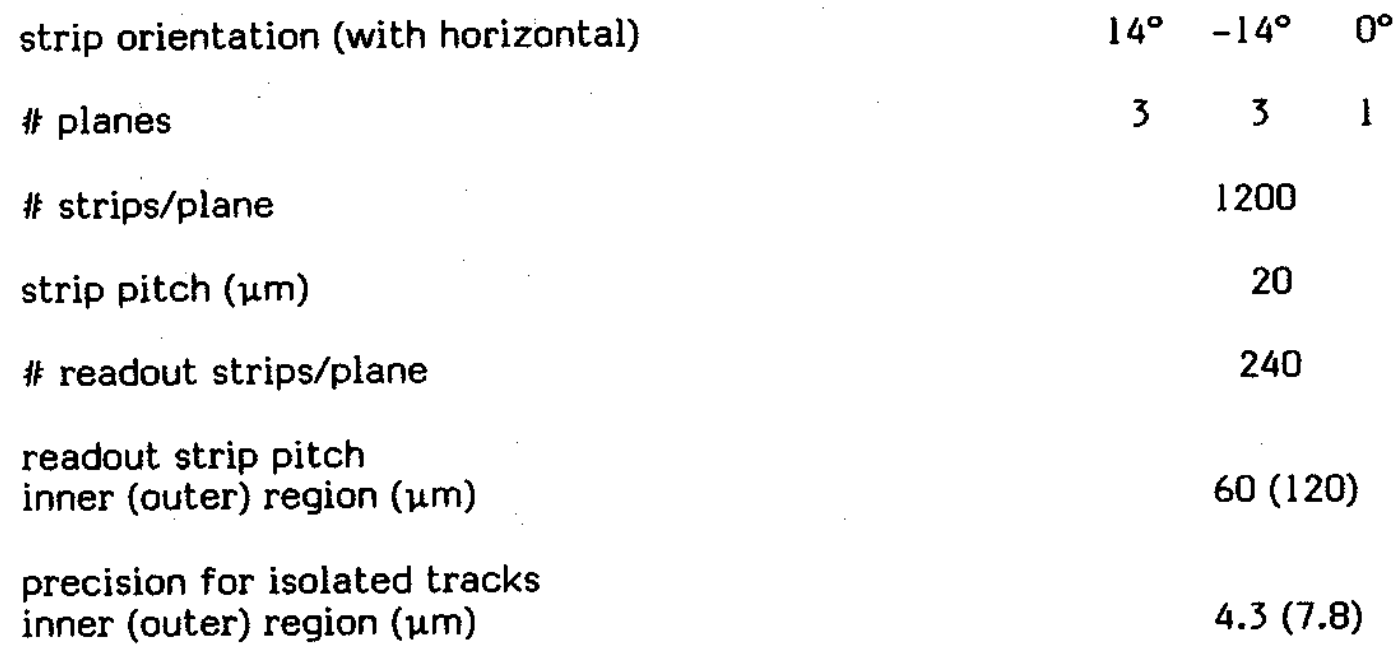

\section{Table 4}

Results of the maximum likelihood fits to the mass spectra and to the lifetimes of the $D$ mesons $\left(\tau_{D}\right)$ and of the background $\left(\tau_{b g}\right)$<smiles>[O+]=[Te]</smiles>

\begin{tabular}{|c|c|c|c|c|}
\hline Decay Channel & $K^{\mp} \pi^{ \pm}$ & $K^{\mp} \pi^{ \pm} \pi^{ \pm} \pi^{\mp}$ & $K^{\mp} \pi^{ \pm}+K^{\mp} \pi^{ \pm} \pi^{ \pm} \pi^{\mp}$ & $K^{\mp} \pi^{ \pm} \pi^{ \pm}$ \\
\hline $\operatorname{Mass}\left[\mathrm{MeV} / \mathrm{c}^{2}\right]$ & $1861.7 \pm 1.4$ & $1863.9 \pm 1.3$ & $1862.7 \pm 1.0$ & $1870.2 \pm 1.0$ \\
\hline No. of D mesons & 58 & 32 & 90 & 59 \\
\hline $\begin{array}{l}\text { No. of background } \\
\text { events } / 10 \mathrm{MeV} / \mathrm{c}^{2}\end{array}$ & 1.9 & 2.2 & 4.1 & 1.5 \\
\hline$\tau_{D}\left[10^{-13} \mathrm{~s}\right]$ & $3.7 \pm 0.6$ & $4.9 \pm \frac{1}{0: 1}$ & $4.2 \pm 0.5$ & $10.9 \pm \frac{1}{1: 9}$ \\
\hline$\tau_{b g}\left[10^{-13} \mathrm{~s}\right]$ & $2.3 \pm 0.4$ & $1.8 \pm 0.3$ & $2.0 \pm 0.2$ & $2.5 \pm 0.5$ \\
\hline
\end{tabular}




\section{Fiqure captions}

Fig. 1 : Schematic layout of the NA32 spectrometer showing the target region $(T)$, magnets (M), drift chambers (DC) and Cerenkov hodoscopes (C).

Fig. 2 : View of the NA32 target region.

Fig. 3: a) Display of an interaction as seen in the active target. The columns depict the detectors, the rows depict the strips. The ADC pulse heights are given in units of 0.1 m.i.p. An overflow is indicated as ****.

b) For the same event we show the tracks as reconstructed by the silicon detector telescope and drift chambers extrapolated back to the target. The active target coordinates (represented as crosses in the figure) are not used in the track fit.

Fig. 4 : Kaon identification efficiency as a function of momentum.

Fig. 5 : Mass spectra for $\mathrm{K}_{\mathrm{s}}^{0}$ with a decay length below $10 \mathrm{~mm}$ a) before and $\mathrm{b}$ ) after rejection on the basis of the pulse height measured in the active target.

Fig. 6: Invariant mass distributions for accepted events. The histogram in Fig. $6 \mathrm{c}$ is the sum of a) and b). The superimposed curves are the result of a fit using the calculated spectrometer resolution.

Fig. 7 : Corrected proper time spectra for the $\mathrm{D}^{\circ}$ and $\mathrm{D}^{+}$candidates. We define the $D$ candidates as those events having an invariant mass in the range $1.83-1.89 \mathrm{GeV} / \mathrm{c}^{2}$ or $1.84-1.90 \mathrm{GeV} / \mathrm{C}^{2}$ for $D^{\circ}$ and $\mathrm{D}^{+}$respectively. The lines show the result of the maximum likelihood fit. The dashed area shows the background contribution in that same mass range.

Fig. 8: a) Distribution of measured flight paths $\ell$ for $K_{\dot{S}}^{\circ}$ The superimposed curve shows the same distribution generated by Monte Carlo.

b) Distribution of measured flight path $\ell$ minus minimum acceptable flight path $\ell^{\min }$ for $K_{s}^{\circ}$. 


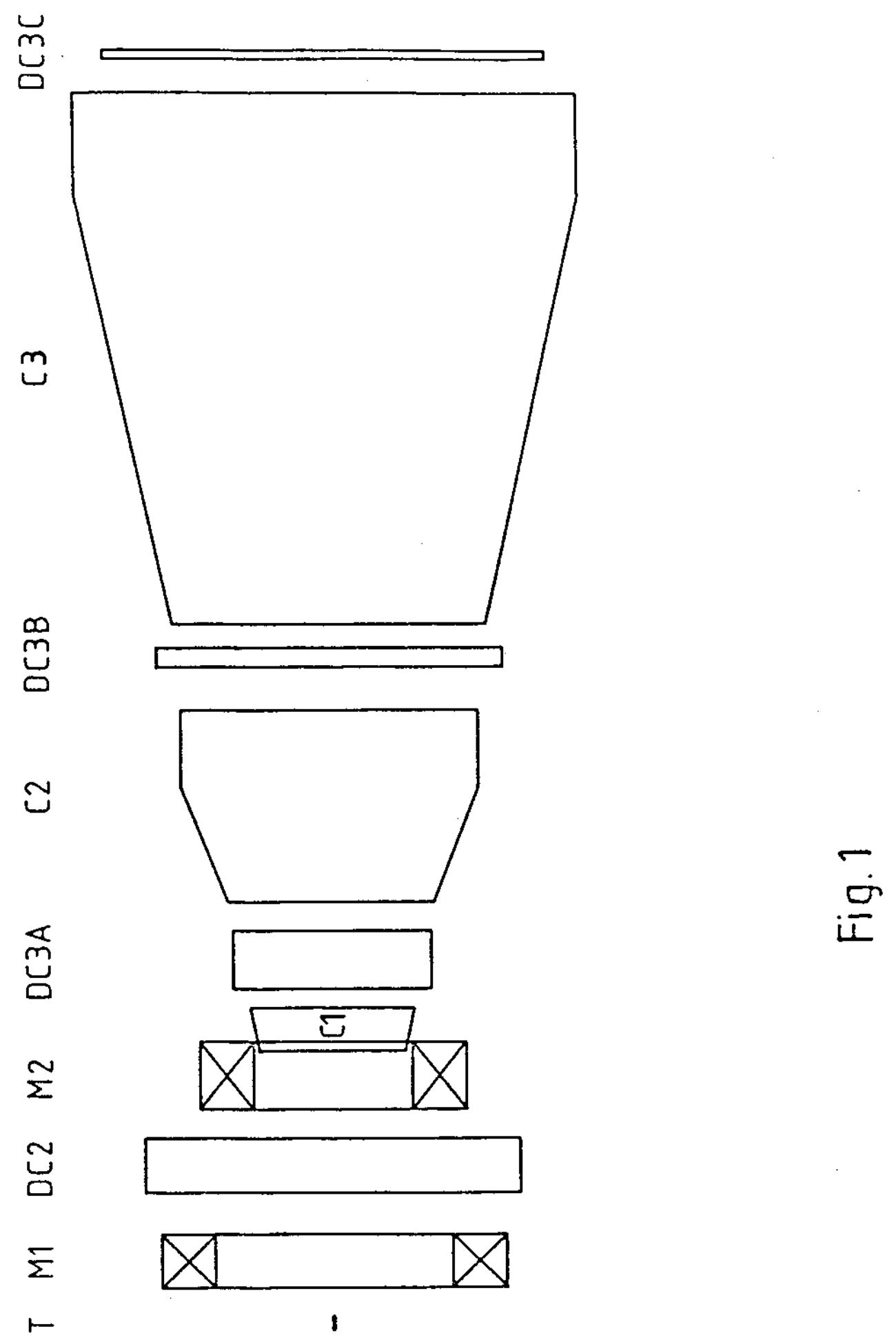

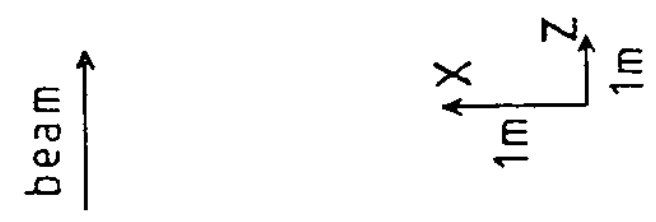




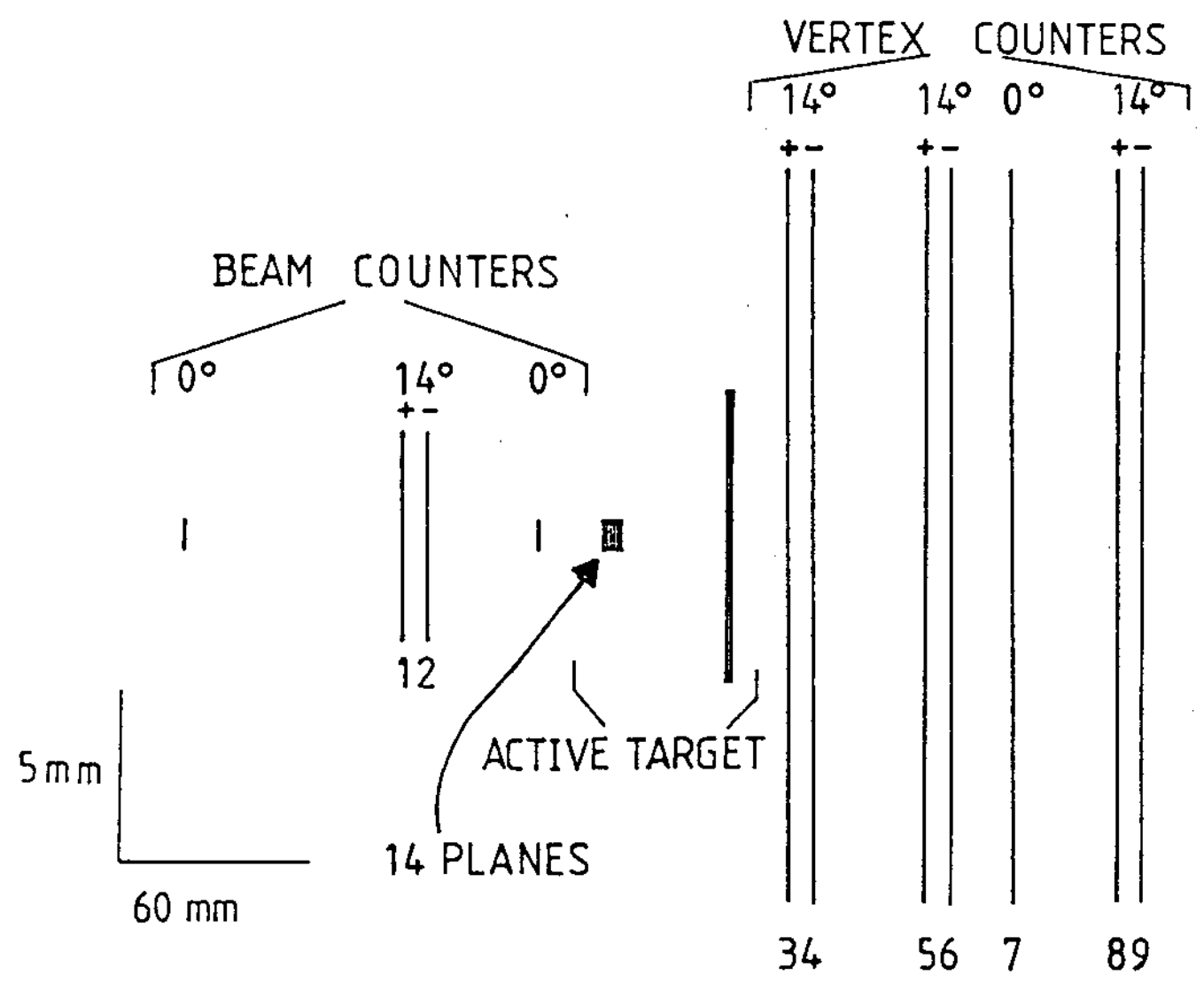

Fig. 2 

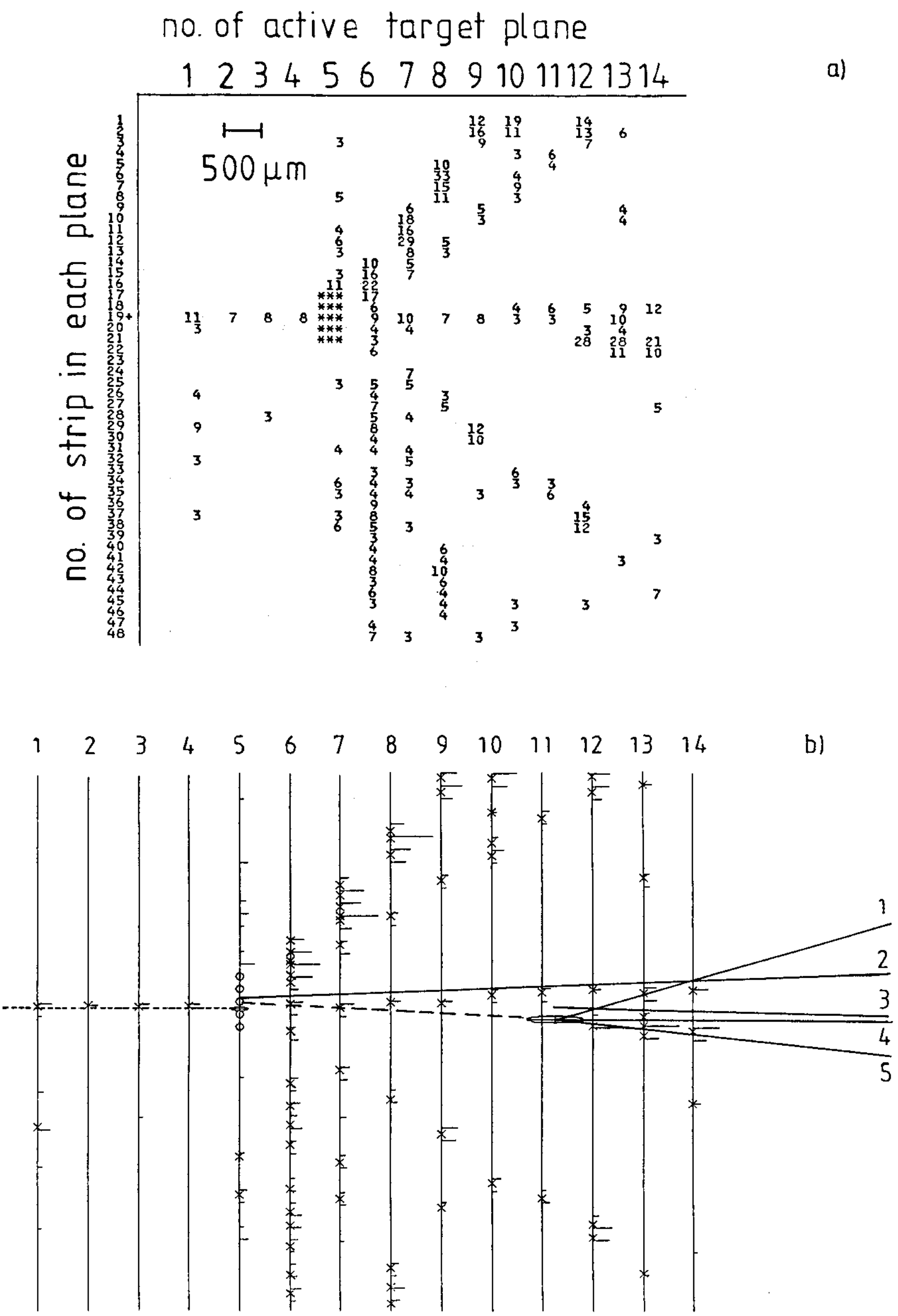

Fig. 3 


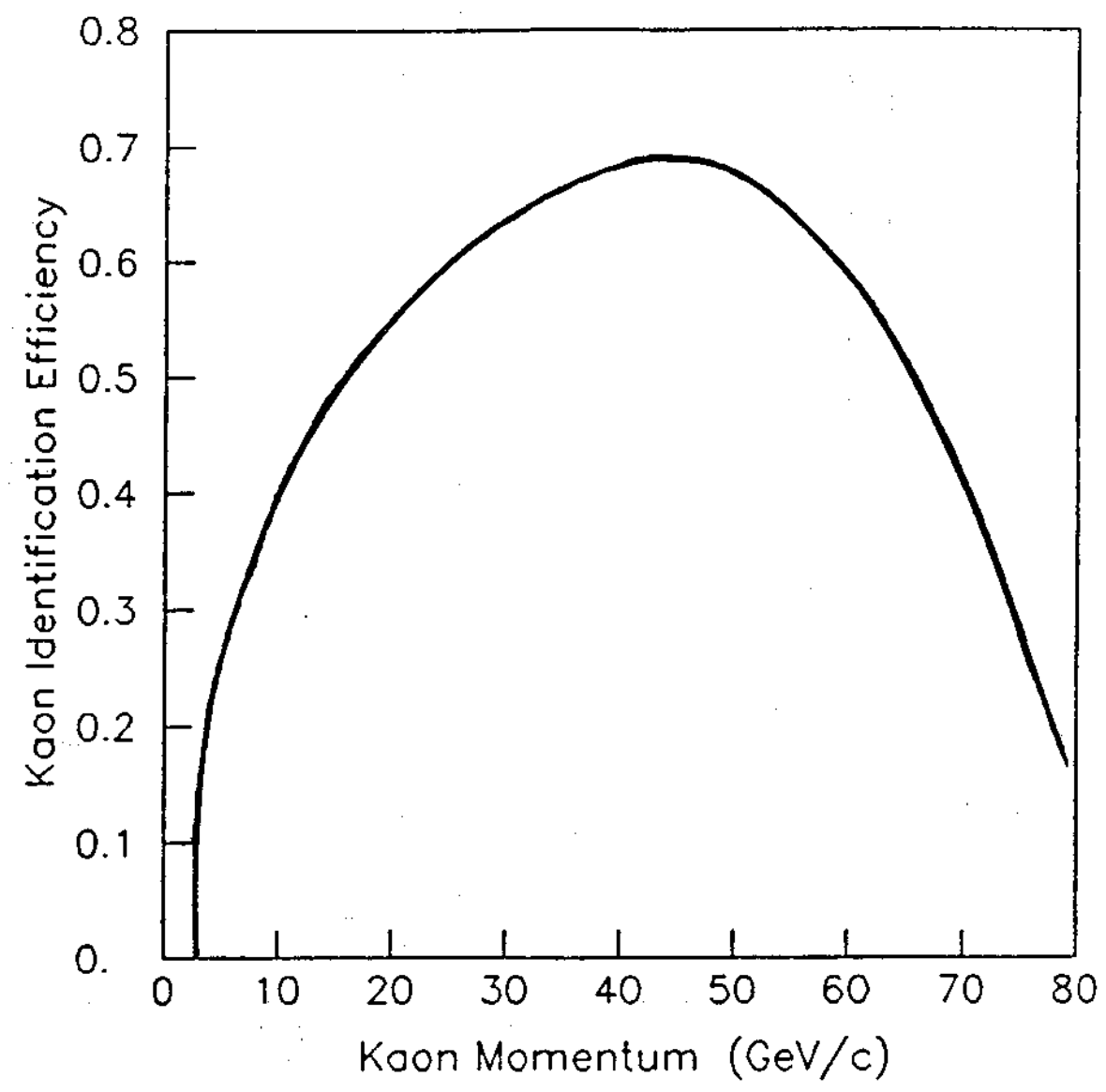

Fig. 4 


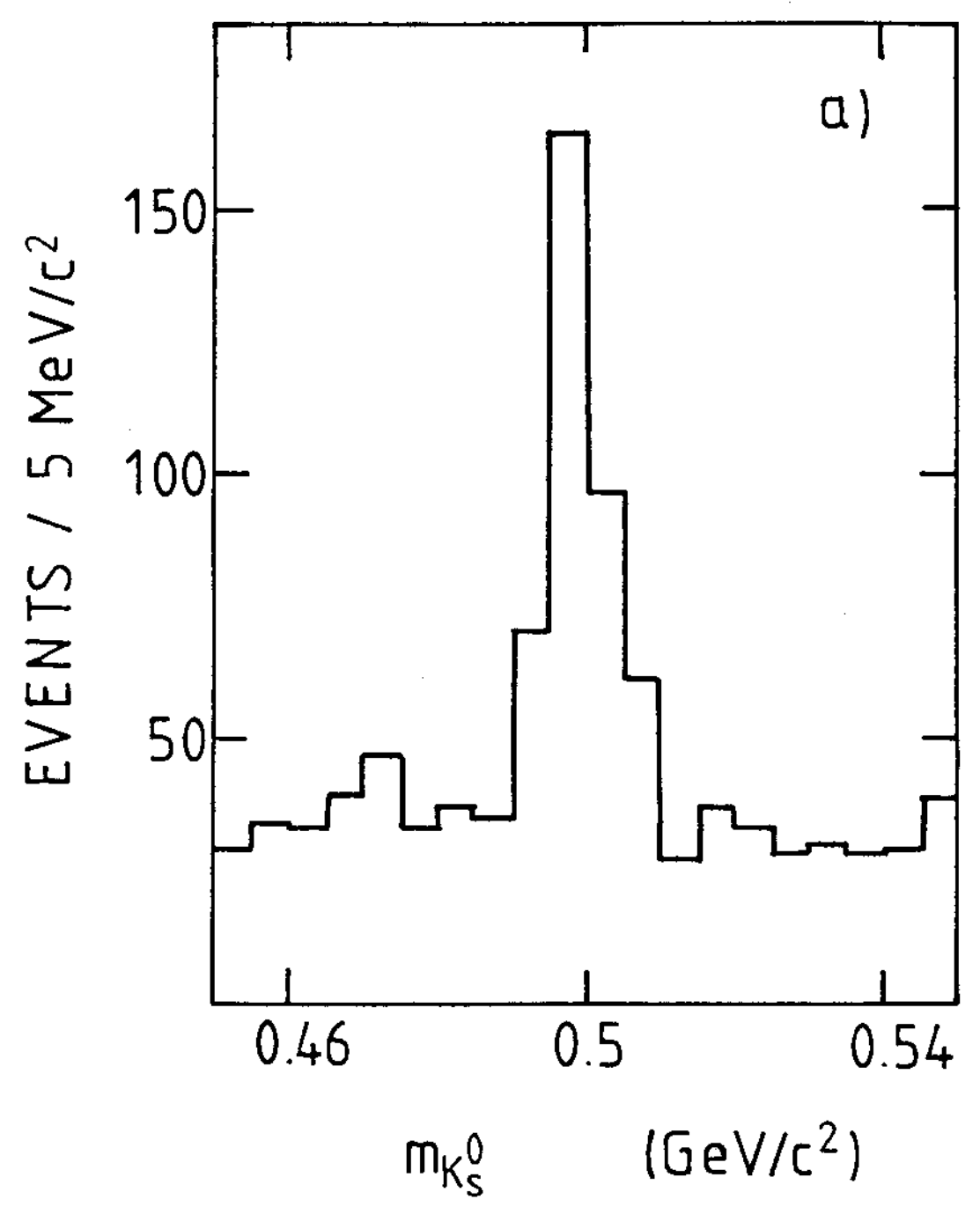

before active target cut

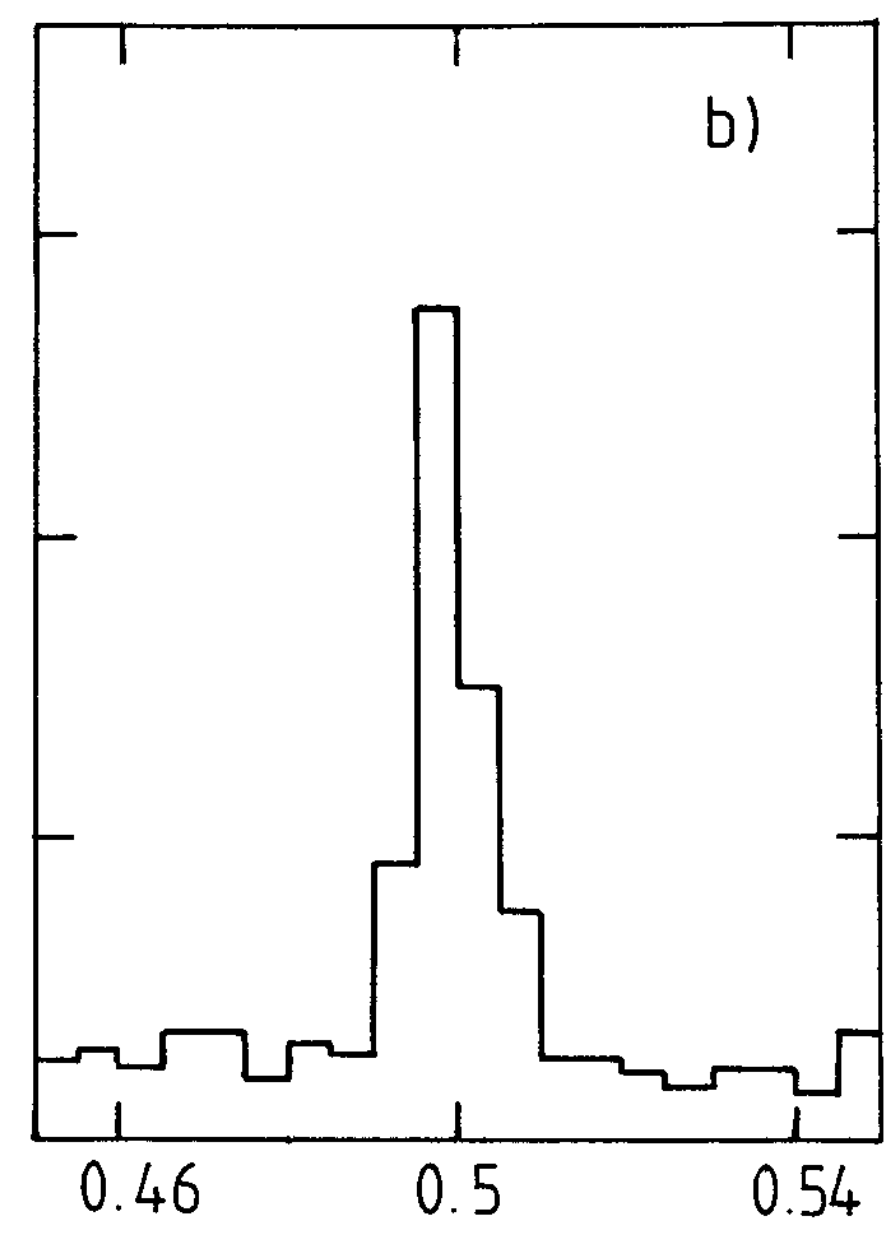

$m_{K_{s}^{0}} \quad\left(G e V / c^{2}\right)$ after active target cut

Fig. 5 

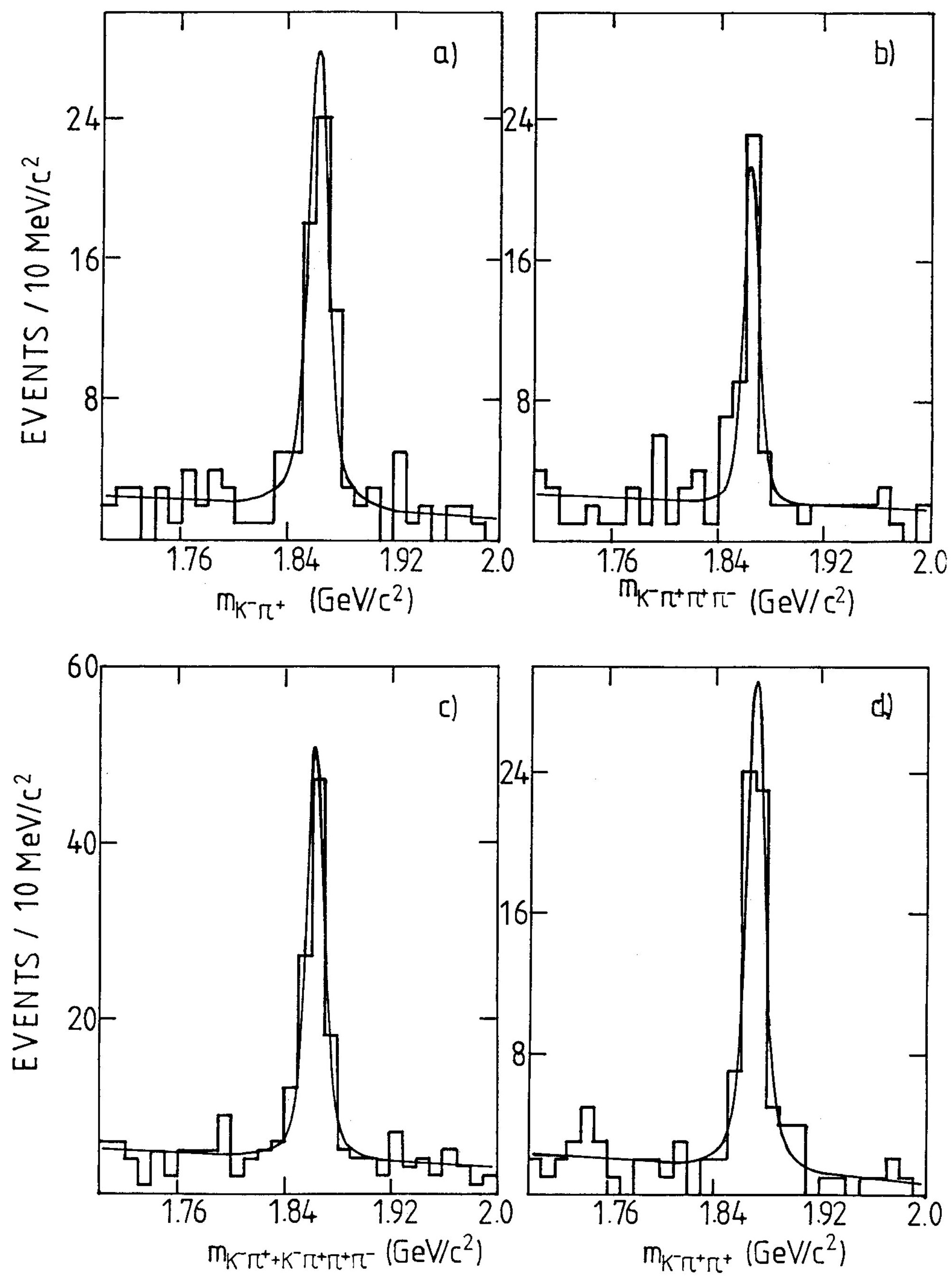

Fig. 6 

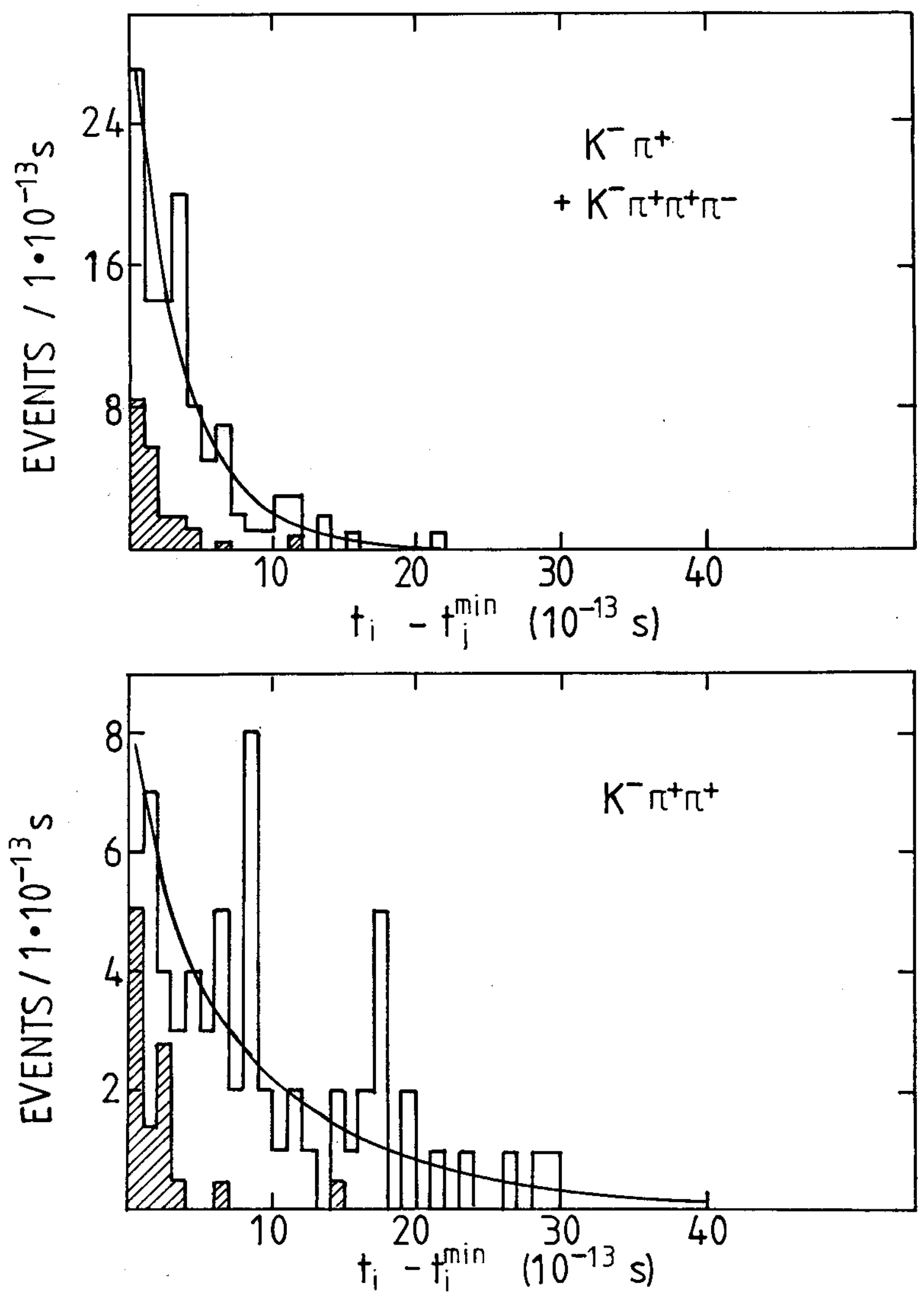

Fig. 7 


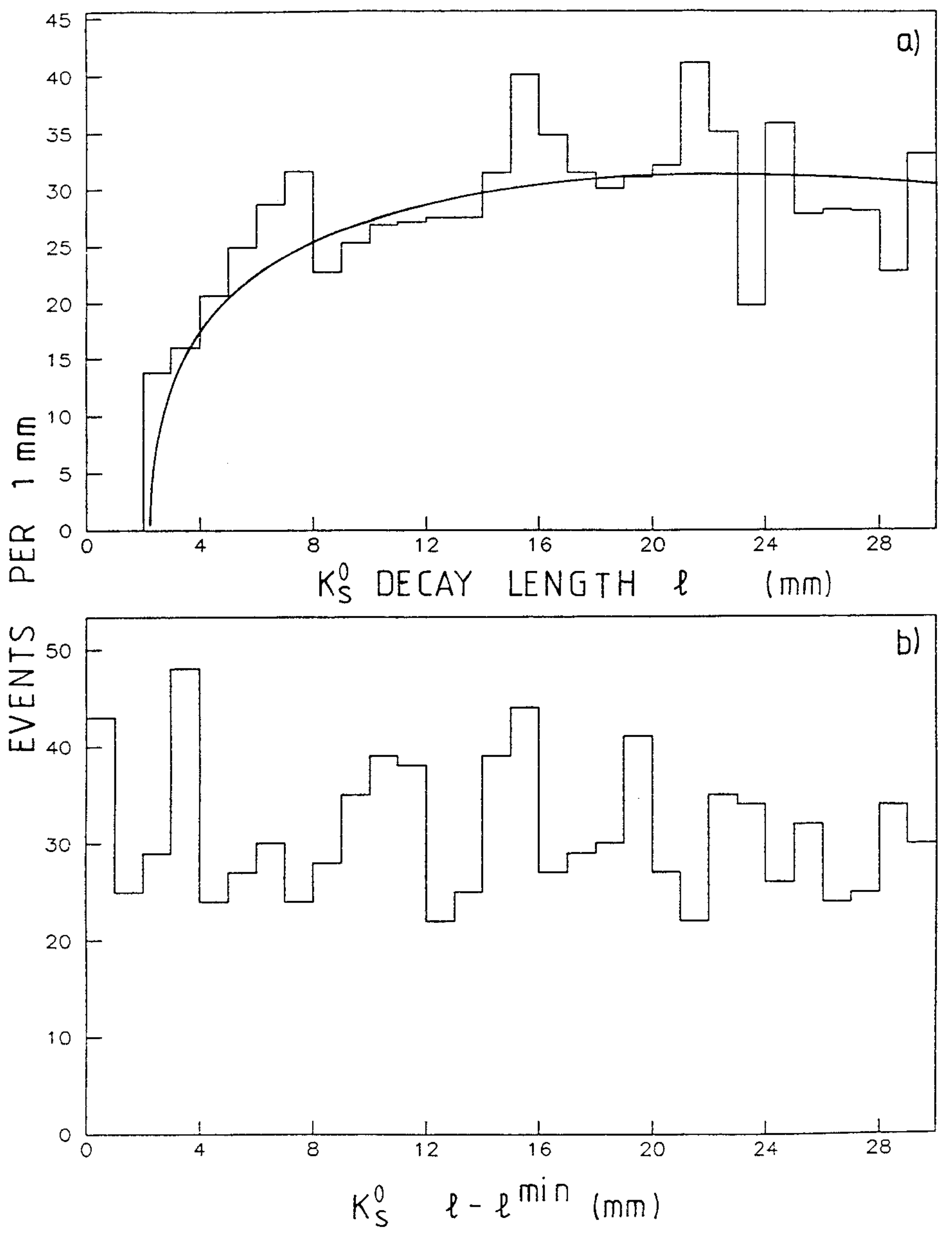

Fig. 8 